\title{
Recovery Phase Spontaneous Nystagmus, Its Existence and Clinical Implication
}

\author{
Min Young Lee ${ }^{1 *}$, Hye Ran Son ${ }^{1 *}$, Yoon Chan Rah ${ }^{2}$, Jae Yun Jung ${ }^{1}$, and Myung-Whan Suh ${ }^{3}$ \\ 1Department of Otorhinolaryngology-Head \& Neck Surgery, Dankook University Hospital, Cheonan, Korea \\ ${ }^{2}$ Department of Otorhinolaryngology-Head \& Neck Surgery, Korea University Ansan Hospital, Ansan, Korea \\ ${ }^{3}$ Department of Otorhinolaryngology-Head \& Neck Surgery, Seoul National University Hospital, Seoul, Korea
}

\author{
Received May 3, 2018 \\ Revised July 16, 2018 \\ Accepted July 31, 2018
}

\section{Address for correspondence Myung-Whan Suh, MD, PhD \\ Department of Otorhinolaryngology- Head \& Neck Surgery, \\ Seoul National University Hospital, \\ 101 Daehak-ro, Jongno-gu, \\ Seoul 03080, Korea \\ Tel +82-2-2072-4845 \\ Fax +82-2-766-2447 \\ E-mail drmung@naver.com \\ *These authors contributed equally to this work.}

Background and Objectives: Determination of the lesion side based on the direction of the nystagmus could result in confusions to the clinicians due to mismatch between the vestibular function tests and also between vestibular and audiologic features. To minimize these mistakes, we elucidated the clinical manifestation and vestibular function test results in cases with recovery spontaneous nystagmus (rSN). Subjects and Methods: Patients who visited ENT clinic of tertiary referral hospital for acute onset continuous vertigo from January 2008 to December 2011 were enrolled. In these patients, we assessed onset time of vertigo, time point of paralytic spontaneous nystagmus (SN) and time point of rSN. At each time point of SN, vestibular function tests and hearing function tests were performed. Results: We confirmed the rSN among patients with unilateral vestibulopathy and demonstrated that high gain of the rotatory chair test (slow harmonic acceleration) and/or mismatch of the SN direction and contralateral caloric weakness could indicate the recovery state of patients and nystagmus observed in this stage is recovery phase nystagmus. Conclusions: In acute vestibulopathy patients, recovery phase nystagmus was observed and on this stage of disease vestibular function tests shows several features that could predict recovery state.

J Audiol Otol 2019;23(1):33-38

KEY WORDS: Spontaneous nystagmus · Recovery spontaneous nystagmus Unilateral vestibulopathy.

\section{Introduction}

Dizziness is a common symptom that patient complains in otology clinics. Its differential diagnosis is based on patient's history and vestibular function test. The majority of vestibular function tests rely on the principle concept of vestibular-ocular reflex. Therefore evaluation of eye movement is essential process in diagnosis of dizziness [1]. Determining the lesion side is not a difficult process in most cases and it is usually based on vestibular function tests. There are multiple ways to assess the lesion side. Among them, most commonly adopted test results are: direction of spontaneous nystagmus (SN; nystagmus in the primary eye position), canal paresis (CP) from ca-

This is an Open Access article distributed under the terms of the Creative Commons Attribution Non-Commercial License (https://creativecommons.org/licenses/by-nc/4.0/) which permits unrestricted non-commercial use, distribution, and reproduction in any medium, provided the original work is properly cited. loric test, and asymmetry evaluated by rotatory chair test [slow harmonic acceleration (SHA)]. Presence of catch-up saccade during head impulse test implicates the lesion side and helps distinguishing the central vertigo. Still, false negative and positive rate exists and does not ensure the complete diagnosis of lesion side or central pathology.

There are some cases that lesion side determined by vestibular function tests is not identical between the tests at different time points. Besides the central nystagmus [2-4] and atypical benign paroxysmal positional vertigo $[5,6]$, most probable explanation for this is recovery spontaneous nystagmus (rSN). rSN usually follows the initial paralytic nystagmus [7]. The direction of nystagmus in recovery phase is known to be ipsilesional which is opposite to the paralytic nystagmus [7]. In other words, "paralytic nystagmus" is the initial nystagmus with the fast phase of nystagmus directed away from the affected ear, and "recovery phase nystagmus" is a reversal of the 
nystagmus with the fast phase directed toward the affected ear. The lesion side prediction based on the rSN could result in confusions to the clinicians due to mismatch between the vestibular function tests and also between vestibular and audiologic features.

Not many reports have been described direction change of nystagmus overtime in peripheral vestibulopathy [8-10]. In the present study, we collected the data from the patients who showed changes of nystagmus direction at two different time point and elucidated the clinical manifestation and vestibular function test results.

\section{Subjects and Methods}

\section{Subjects}

We retrospectively reviewed the data of the patients who visited ENT clinic of Dankook University Hospital for acute onset (first attack) continuous ( $>24 \mathrm{hrs}$ ) vertigo from January 2008 to December 2011. Only the patients with symptoms and sign of acute unilateral vestibular weakness and with both paralytic SN and rSN are included in the study. The patients with vertigo suspected of central pathology, bilateral vestibular weakness and unclear catch up saccade in head impulse test are excluded from the study. This study has been approved by the Ethical Committee of Faculty of Medicine, Dankook University Hospital (2016-08-007-004).

\section{Clinical manifestation}

We assessed onset time of vertigo, time point of paralytic SN and time point of rSN. At each time point of SN, vestibular function teats and hearing function tests were performed. To evaluate the vestibular function videonystagmography (VNG), caloric test and rotation chair tests were performed. SN with and without visual fixation was recorded with a System 2000 VNG (Micromedical, Chatham, IL, USA). An alternate binaural bithermal caloric test was performed with $30^{\circ} \mathrm{C}$ cool and $44^{\circ} \mathrm{C}$ warm water. On the rotating chair test with the System 2000 Rotation Chair System (Micromedical), eye movements were recorded. Patients' hearing functions are evaluated with pure tone audiometry and hearing thresholds were measured at both paralytic and recovery phase. Aurical Plus (GN Otometrics, Taastrup, Denmark) was used and frequencies from $0.5 \mathrm{kHz}$ to $8 \mathrm{kHz}$ were assessed.

\section{Results}

\section{Demographic data and information of nystagmus}

Mean age of the included subjects were $55.1 \pm 14.4$ years. The study group showed female predominance $(\mathrm{M}: \mathrm{F}=5: 11)$.
Patients visited the clinic $6.94 \pm 6.47$ days after onset of symptom. Initial nystagmus which suggests paralytic nystagmus was detected at $7.25 \pm 11.85$ days after the onset of dizziness and following direction changed nystagmus which suggests recovery nystagmus was detected $17.56 \pm 14.16$ days (4-56 days) after the onset of dizziness (Fig. 1). Most of rSN (81.25\%) was found within 1 month. The time gap between the two nystagmus was $10.25 \pm 9.45$ days. Twelve out of 14 patients showed unilateral vestibular hypofunction determined either by caloric or SHA test. No dramatic change of symptoms was notified at the point of recovery nystagmus.

Eight subjects showed initial left beating nystagmus indicating the right vestibulopathology, at the recovery phase directions of these nystagmus were changed (right beating) (Fig. 2 ). The other seven subjects showed initial right beating nystagmus indicating the left vestibulopathology at the recovery phase directions of these nystagmus were changed (left beating). Directions of nystagmus were not altered after provocation by head shake in both paralytic and recovery phases (Table 1). These results confirm the presence of rSN among patients with unilateral vestibulopathy.

\section{Results of the vestibular function tests}

Total 16 patients showed the different direction of nystagmus at two different time point and at each time point direction of nystagmus were not altered after provocation by head shake (Table 1). Vestibular function tests such as SHA and caloric test were performed to objectively assess the gain and lesion side. Fourteen patients were tested with SHA and 12 patients were tested with caloric test. In the analysis of SHA test, most of patients revealed low gain and ipsilesional weakness at paralytic phase. However at the recovery phase six patients showed normal to high gain and all patients showed normal or contralesional asymmetry (Fig. 3, Table 2). In five subjects, SHA tests were performed in both phases, the two test results were different in all cases. The low gain were changed to normal to high gain in three subjects and a patient showed change from normal gain to low gain. The ipsilesional weak-

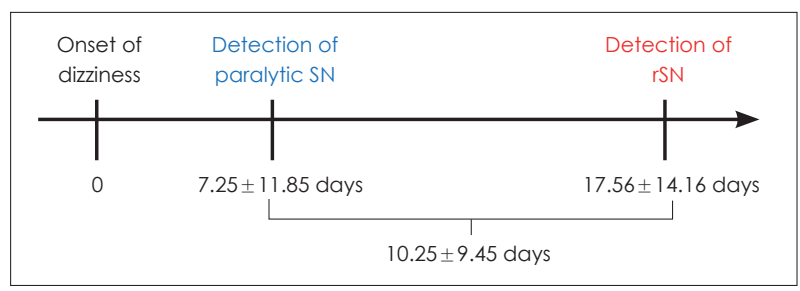

Fig. 1. Time points of paralytic $\mathrm{SN}$ and $\mathrm{rSN}$ detection. Paralytic SN was observed at $7.25 \pm 11.85$ days from the onset of dizziness and rSN was observed at $17.56 \pm 14.16$ days from the onset of dizziness. SN: spontaneous nystagmus, rSN: recovery spontaneous nystagmus. 


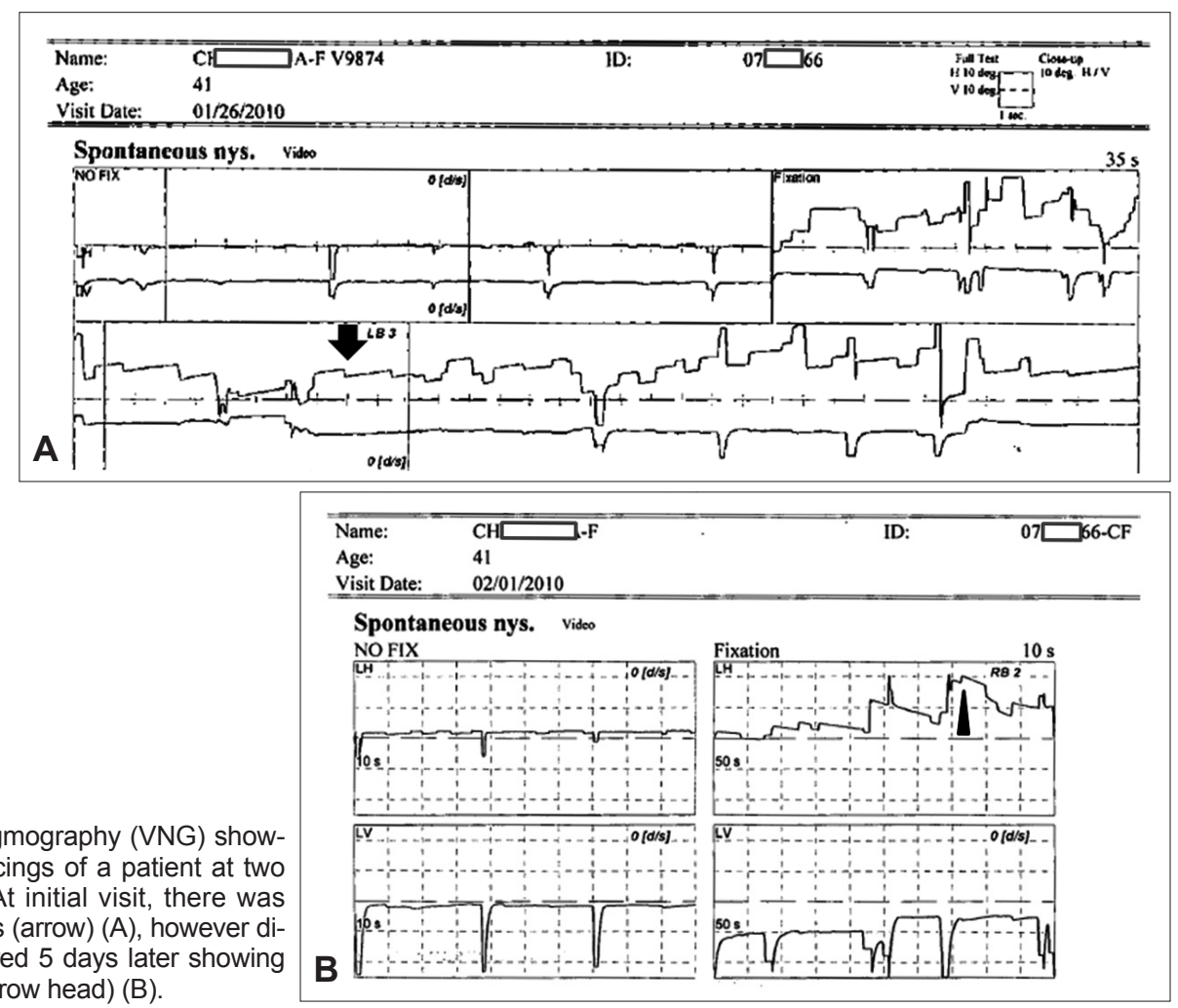

Fig. 2. Representative videonystagmography (VNG) showing recovery nystagmus. VNG tracings of a patient at two different time point are showed. At initial visit, there was spontaneous left beating nystagmus (arrow) (A), however direction of this nystagmus was altered 5 days later showing spontaneous nystagmus to right (arrow head) (B).

Table 1. Patient information and direction

\begin{tabular}{|c|c|c|c|c|c|c|}
\hline & \multirow{2}{*}{ Sex/Age } & \multirow{2}{*}{ Lesion side } & \multicolumn{2}{|c|}{ SN } & \multicolumn{2}{|c|}{ HSN } \\
\hline & & & Paralytic & Recovery & Paralytic & Recovery \\
\hline P01 & $M / 64$ & Rt. & LB & $\mathrm{RB}$ & LB & - \\
\hline P02 & $F / 41$ & Rt. & LB & $R B$ & LB & RB \\
\hline P03 & $\mathrm{F} / 57$ & Rt. & LB & RB & LB & RB \\
\hline P04 & $F / 57$ & Rt. & LB & $R B$ & LB & RB \\
\hline P05 & $\mathrm{F} / 75$ & Rt. & LB & RB & LB & RB \\
\hline P06 & $F / 33$ & Rt. & LB & RB & LB & $R B$ \\
\hline P07 & $F / 41$ & Rt. & LB & $R B$ & LB & $R B$ \\
\hline P08 & $M / 71$ & Rt. & LB & $R B$ & LB & $R B$ \\
\hline P09 & $M / 40$ & Rt. & LB & $R B$ & LB & RB \\
\hline P10 & $M / 42$ & Lt. & $R B$ & LB & RB & - \\
\hline P11 & M/85 & Lt. & $R B$ & LB & $R B$ & LB \\
\hline $\mathrm{P} 12$ & $\mathrm{~F} / 45$ & Lt. & RB & LB & RB & LB \\
\hline P13 & $F / 64$ & Lt. & $\mathrm{RB}$ & LB & $R B$ & LB \\
\hline $\mathrm{P} 14$ & $F / 56$ & Lt. & $R B$ & LB & $R B$ & LB \\
\hline P15 & $F / 51$ & Lt. & $\mathrm{RB}$ & LB & $R B$ & LB \\
\hline P16 & $\mathrm{F} / 60$ & Lt. & $\mathrm{RB}$ & LB & $\mathrm{RB}$ & LB \\
\hline
\end{tabular}

SN: spontaneous nystagmus, HSN: post-head shake nystagmus, LB: left beating nystagmus, RB: right beating nystagmus, -: not performed

ness was changes to contralesional weakness in three subjects and a patient showed change from ipsilesional to normal asymmetry (Table 2). On the contrary, caloric test mostly showed ipsilesional weakness in both paralytic and recovery phases. Three patients have gone through caloric test in both phases. Among these patients, two subjects showed aggravation of CP from normal to ipsilesional weakness (Table 2).

\section{Discussion}

$\mathrm{SN}$ is generated due to the difference of tonic resting activity between the vestibular systems (right and left) [10]. The 


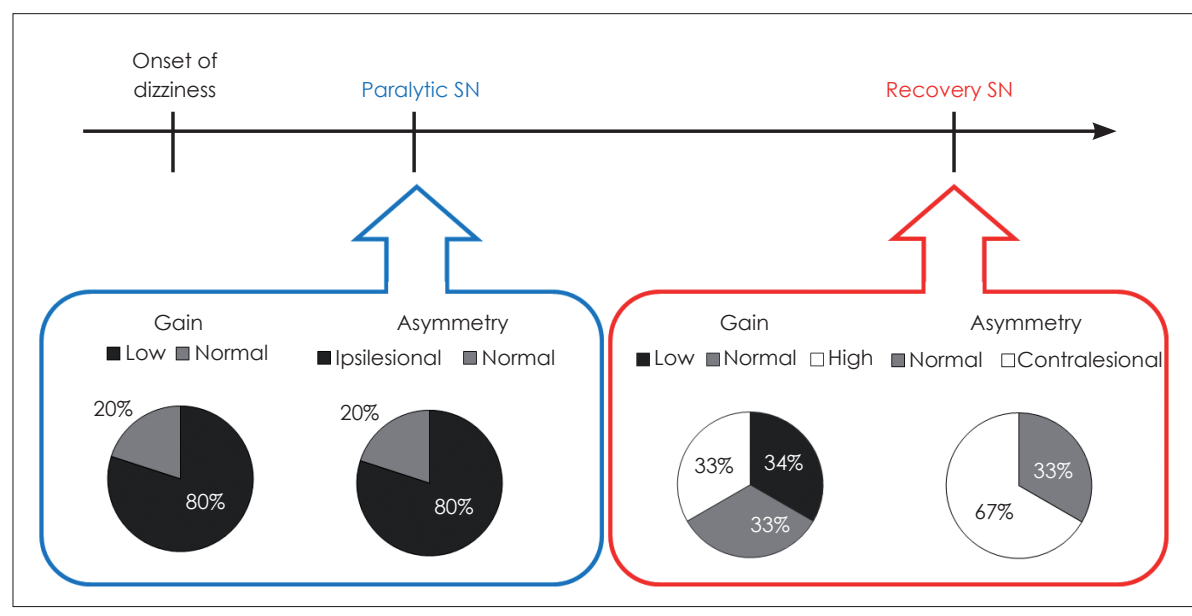

Fig. 3. Rotation chair test (SHA) results. Rotation chair test results altered with disease progression. Majority of the patients (80\%) showed low gain and ipsilesional asymmetry on SHA test at paralytic SN stage. However, at the recovery phase, $66 \%$ of patients showed normal or high gains and none showed ipsilesional asymmetry. In addition, at this stage, $67 \%$ of patients showed contralesional asymmetry. SHA: slow harmonic acceleration.

Table 2. The result of SHA and caloric test

\begin{tabular}{|c|c|c|c|c|c|c|}
\hline & \multicolumn{4}{|c|}{ SHA } & \multicolumn{2}{|c|}{ Caloric test (CP\%) } \\
\hline & \multicolumn{2}{|c|}{ Paralytic } & \multicolumn{2}{|c|}{ Recovery } & \multirow{2}{*}{ Paralytic } & \multirow{2}{*}{ Recovery } \\
\hline & Gain & Asymmetry & Gain & Asymmetry & & \\
\hline P01 & Low & Ipsilesional & - & - & Ipsilesional weak (66) & - \\
\hline P02 & Normal & Normal & Low & Contralesional & Ipsilesional weak (28) & - \\
\hline P03 & - & - & Low & Contralesional & - & Ipsilesional weak (50) \\
\hline P04 & - & - & - & - & - & Ipsilesional weak (33) \\
\hline P05 & - & - & Normal & Normal & - & Normal (14) \\
\hline P06 & Low & Ipsilesional & High & Contralesional & Ipsilesional weak (26) & Ipsilesional weak (72) \\
\hline P07 & Low & Ipsilesional & Low & Contralesional & Normal (7) & Ipsilesional weak (29) \\
\hline P08 & Low & Ipsilesional & High & Contralesional & Normal (8) & Ipsilesional weak (30) \\
\hline P09 & - & - & High & Contralesional & - & Ipsilesional weak (27) \\
\hline $\mathrm{P} 10$ & Low & Ipsilesional & - & - & Ipsilesional weak (49) & - \\
\hline P11 & Low & Ipsilesional & - & - & - & - \\
\hline $\mathrm{P} 12$ & Low & Ipsilesional & Normal & Normal & - & Ipsilesional weak (25) \\
\hline P13 & - & - & - & - & - & Ipsilesional weak (30) \\
\hline $\mathrm{P} 14$ & Low & Ipsilesional & - & - & Ipsilesional weak (27) & - \\
\hline P15 & - & - & Normal & Normal & - & - \\
\hline $\mathrm{Pl} 6$ & Normal & Normal & - & - & - & - \\
\hline
\end{tabular}

SHA: slow harmonic acceleration, CP: canal paresis, -: not performed

precise amount of tonic difference to generate the $\mathrm{SN}$ is not known but usually $\mathrm{SN}$, fast eye movement; direction is away from the lesion side [11]. But occasionally SN towards the lesion side is observed in clinic, this could be explained by two possible theories. Ipsilesional nystagmus could be observed in the pathologies that increases neural firing rate. This 'irritative nystagmus' could be observed [7], though rare, in early stage of Meniere's disease by chronic depolarization of hair cells due to hydrops induced mechanical deflection of lateral semicircular canal cupulae. Alternatively this ipsilesional nystagmus could be observed in the recovery phase of vestibulopathies [9]. Recovery nystagmus is believe to be related to the serial compensation of peripheral vestibular system by central nervous system, which is described in detail on the prior document by Jacobson, et al. [10]. To explain this mechanism in simple way, it can be explained as earlier compensatory increase of ipsilesional tonic activity compared to the contralesional regulated tonic activity. This observation was thought to be important since it proved functional recovery of affected lesion after vestibulopathy $[9,12]$. Nowadays due to relative short observation time of patients and high price of vestibular function tests, serial assessment of nystagmus is not feasible. Furthermore possibility of misdiagnosis of lesion side by observation of this recovery nystagmus is underestimated. This study proves the change of nystagmus direction documented with VNG suggesting the existence of rSN. 
Table 3. Clinical observations to consider rSN

Clinical finding to consider rSN
Nystagmus observed at delayed time point
Mismatch of SN direction with CP value of caloric test
High gain with contralesional asymmetry in SHA test

rSN: recovery spontaneous nystagmus, CP: canal paresis, SHA: slow harmonic acceleration of SN and HSN

When confronting the dizziness patients with $\mathrm{SN}$ in the clinic, we should be able to distinguish the paralytic nystagmus from recovery nystagmus. Followings are the several points from the present study which might help this process (Table 3). First thing is the onset of the disease, according to our result it is found that recovery nystagmus was observed at 17 days after onset which is 10 days later from the observation of paralytic nystagmus. This result provides the evidence that if the nystagmus were observed at delayed time point we have to suspect the recovery nystagmus.

Secondly we can rely on $\mathrm{CP}$ value for this differential diagnosis. Regardless of the time point of the test, ipsilesional weakness of $\mathrm{CP}$ value was found in most the patients (13 out of 16 tests). Therefore, in case of mismatch of SN direction with $\mathrm{CP}$ value of caloric test, $\mathrm{rSN}$ has to be suspected and we should decide the lesion side according to the weak side of $\mathrm{CP}$ value.

Last information could be obtained from the SHA test. Among the parameters of SHA test, asymmetry was not reliable parameter in deciding the lesion sides since the directions of asymmetry were changed concurrently with the SN direction. Therefore deciding the lesion side according the weakness side of asymmetry could lead to the misdiagnosis. On the contrary, several patients with recovery nystagmus showed high gain with contralesional asymmetry. The reason for this is unclear, we still do not know whether this result is from the over compensation from the central nervous system or recovery of the peripheral nervous system. However, this could be a good marker for recovery phase and asymmetry direction observe at high gain stage might be contralesional side as well as rSN. In the cases with the mismatched lesion side results, our results might provide additional information to help determination of the lesion side.

According to the previous report by Matsuzaki, et al. [7], it is known that recovery nystagmus induced by head shake test precedes the rSN. Therefore, in the early stage of recovery, ipsilesional nystagmus induced by head shake test could be observed. In this study, however, these findings were not observed and all of the headshake induced nystagmus was toward the contralesion side. These outcomes suggest that the time points for the VNG in this study is adequate to represent the both par- alytic and recovery stage and are not too early or too late.

In the current study, we have observed the recovery phase spontaneous nystagmus, direction of the nystagmus changed about 10 days after initial nystagmus. According to the comparison of the several vestibular function tests which help the determination of the lesion side, we found out that high gain of the SHA and mismatch of the SN direction of CP paresis weak side could indicate the rSN. With the result of this study, we are raising a possible explanation for the mismatches of the lesion side between the tests which clinicians encounter in the diagnosis of the vestibulopathy. Further analysis adopting other functional test which could predict lesion side such as vestibular evoked myogenic potential, video head impulse test and measurement of subject visual vertical/horizontal [13] will provide more information that could help the lesion side prediction and rSN. There are several things to consider before planning the further analysis of the current data. First, female predominance should be assessed. Small number of subjects limits the further analysis but the hormonal issue might be related to observation of rSN. Reports showing the relation of hormonal receptor and dizziness [14], and effect of hormonal replacement therapy for menopause induced dizziness [15] supports the idea. Enrolled subjects of current study could be a composite of heterogenic disease population. Patients with audiologic symptoms such as earfullness and hearing loss are included and future development of Meniere's disease is possible among these subjects. Further study should exclusively be focused on the vestibulopathy patients and Meniere's disease respectively.

\section{Acknowledgments}

This research was supported by the Korea Health Industry Development Institute (KHDI) of Korean Ministry of Health and Welfare (HI18C0626).

\section{Conflicts of interest}

The authors have no financial conflicts of interest.

\section{REFERENCES}

1) King WM. Getting ahead of oneself: anticipation and the vestibuloocular reflex. Neuroscience 2013;236:210-9.

2) Cnyrim CD, Newman-Toker D, Karch C, Brandt T, Strupp M. Bedside differentiation of vestibular neuritis from central "vestibular pseudoneuritis." J Neurol Neurosurg Psychiatry 2008;79:458-60.

3) Mandalà M, Nuti D, Broman AT, Zee DS. Effectiveness of careful bedside examination in assessment, diagnosis, and prognosis of vestibular neuritis. Arch Otolaryngol Head Neck Surg 2008;134:164-9.

4) Newman-Toker DE, Kattah JC, Alvernia JE, Wang DZ. Normal head impulse test differentiates acute cerebellar strokes from vestibular neuritis. Neurology 2008;70(24 Pt 2):2378-85.

5) Chang YS, Choi J, Chung WH. Persistent direction-fixed nystagmus following canalith repositioning maneuver for horizontal canal BPPV: a case of canalith jam. Clin Exp Otorhinolaryngol 2014;7:138-41.

6) Lee HJ, Kim YH, Hong SK, Kim HJ. Pseudo-spontaneous nystag- 
mus in lateral semicircular canal benign paroxysmal positional vertigo. Clin Exp Otorhinolaryngol 2012;5:201-6.

7) Matsuzaki M, Kamei T. Stage-assessment of the progress of continuous vertigo of peripheral origin by means of spontaneous and headshaking nystagmus findings. Acta Otolaryngol Suppl 1995;519:188-90.

8) Stenger HH. ["Recovery nystagmus" after unilateral vestibular prolapse, a process related to Bechterew nystagmus]. Arch Ohren Nasen Kehlkopfheilkd 1959;175:545-9.

9) McClure JA, Lycett P. Recovery nystagmus. J Otolaryngol 1978;7: 141-8.

10) Jacobson GP, Pearlstein R, Henderson J, Calder JH, Rock J. Recovery nystagmus revisited. J Am Acad Audiol 1998;9:263-71.

11) Yagi $T$. Nystagmus as a sign of labyrinthine disorders--three-dimensional analysis of nystagmus. Clin Exp Otorhinolaryngol 2008;1:63-74.
12) Parnes LS, McClure JA. Rotatory recovery nystagmus: an important localizing sign in endolymphatic hydrops. J Otolaryngol 1990;19: 96-9.

13) Kim KW, Lee MY, Jung JY. Mastoid vibration reduces ipsilesional shift of subjective visual horizontal in patients with acute stage of unilateral vestibulopathy. J Audiol Otol 2017;21:66-71.

14) Lee H, Sininger L, Jen JC, Cha YH, Baloh RW, Nelson SF. Association of progesterone receptor with migraine-associated vertigo. Neurogenetics 2007;8:195-200.

15) Coksuer H, Koplay M, Oghan F, Coksuer C, Keskin N, Ozveren O. Effects of estradiol-drospirenone hormone treatment on carotid artery intima-media thickness and vertigo/dizziness in postmenopausal women. Arch Gynecol Obstet 2011;283:1045-51. 